\title{
Inadvertent Postoperative Hypothermia at Post-Anesthesia Care Unit: Incidence, Predictors and Outcome*
}

\author{
Clara Luís ${ }^{1}$, Carlos Moreno ${ }^{1}$, Acácio Silva ${ }^{1}$, Rosália Páscoa ${ }^{1}$, Fernando Abelha ${ }^{1,2 \#}$ \\ ${ }^{1}$ Department of Anesthesiology, Centro Hospitalar de São João, Porto, Portugal; ${ }^{2}$ Anesthesiology and Perioperative Care Unit, \\ Surgical Department, Faculty of Medicine of Porto, University of Porto, Porto, Portugal. \\ Email: "fernando.abelha@gmail.com
}

Received September $3^{\text {rd }}, 2012$; revised October $10^{\text {th }}, 2012$; accepted October $20^{\text {th }}, 2012$

\begin{abstract}
Background: Inadvertent postoperative hypothermia (IPH) is known to be associated with various adverse effects. The aim of this study was to evaluate the incidence, predictors and outcome of core inadvertent hypothermia on admission in the post-anesthesia care unit. Methods: Observational, prospective study in a Post-Anesthesia Care Unit. The study population consisted of adult patients after non-cardiac and non-neurologic surgery. Patients' demographics, intraoperative and postoperative data were collected. Descriptive analysis of variables was used to summarize data and the Mann-Whitney U test, Fisher's exact test or Chi-square test was used. Univariate and multivariate analyses were done with logistic binary regression with calculation of an Odds Ratio (OR) and its 95\% Confidence Interval. Results: The incidence of IPH on admission was 32\%. In univariate analysis: age, body mass index (BMI), high risk surgery, revised cardiac risk index (RCRI), type of anesthesia, use of forced-air warming, amount of intravenous crystalloids administrated, duration of anesthesia, duration of surgery and admission visual analogue scale (VAS) for pain $>3$ were considered predictors of hypothermia. In multiple logistic regression analysis, age (OR 1.7, $\mathrm{P}=0.045$, for age $>65$ years), RCRI (OR 3.18, $\mathrm{P}=0.041$, for RCRI $>2$ ), duration of anesthesia $(\mathrm{OR} 1.52, \mathrm{P}<0.001$ ) and admission VAS for pain (OR 2.05, P $=0.007)$ were considered independent predictors of IPH. Patients with IPH at PACU admission stay longer in the PACU. Conclusions: IPH was associated with a longer stay in the PACU. Age, comorbidities duration of anesthesia and pain at PACU admission were considered independent predictors for IPH.
\end{abstract}

Keywords: Core Temperature; Inadvertent Postoperative Hypothermia; Outcome; Post-Anesthesia Care Unit

\section{Introduction}

Hypothermia is defined as core body temperature less than $36.0^{\circ} \mathrm{C}$, although some studies have considered lower limits because of a high incidence of patients bellow $36^{\circ} \mathrm{C}$ [1-3]. Inadvertent postoperative hypothermia (IPH) emerges as the most common thermic alteration associated with anesthesia and surgery [4]. Despite of core temperature $(\mathrm{Tc})$ normally be tightly controlled in human beings, general anesthesia significantly impairs the thermoregulatory system $[5,6]$, and the same happens with neuraxial (spinal and epidural) anesthesia, even with a potential lesser extent. The result is that unwarmed surgical patients almost inevitably become hypothermic [7]. IPH is a recognized side effect of general and regional anesthesia, once normal thermoregulation is inhibited [2], but Tc is seldom monitored in clinical practice [8].

\footnotetext{
"Funding: authors disclose any financial competing interests but also any non-financial competing interests.

"Corresponding author.
}

IPH is known to be associated with various adverse physiologic effects $[1,9,10]$. Hypothermia alters the normal physiology of most body systems and therefore will also be altered pharmacokinetics and pharmacodynamics of drugs during this state $[3,11]$. Core hypothermia may explain situations as broad as adverse myocardial outcomes in high-risk patients, significantly augment of blood transfusion and surgical wound infection [8,11-13], delayed post anesthetic recovery [12], postoperative negative nitrogen balance [6], increased mortality in elderly patients with sepsis [14], and, with questionable clinical significance, alterations on pulse oximetry monitoring and on various electrophysiological indices of nervous system [3].

Result of an altered distribution of body heat, rather than an imbalance between production and heat loss [4], hypothermia appears when warming techniques are insufficient to counteract thermal redistribution resulting from the ablation of thermoregulatory vasoconstriction associated with anesthesia [15].

Several risk factors are described in literature for the 
development of IPH. The advanced age, sex, low BMI and body surface area, preoperative body temperature, history of diabetic neuropathy, emergency surgery, American Society of Anesthesiology (ASA) physical status, surgical procedure in which major body cavities or major vessels are exposed, anesthetic technique, warming method, amount and temperature of intravenous replacement, duration of anesthesia or surgery and ambient operating room temperature are the risk factors that gather more consensus for many years $[1,2,12,16]$. Risk factors are not independent, and combinations of risk factors may be significant. It may also be important to distinguish between factors that make the patient more likely to become hypothermic, and those that place the patient at greater risk of prolonged hypothermia and/or at greater risk of complications from hypothermia because of their inability to recover from the hypothermic state [2].

Although risk factors are identified, it is thought that perhaps these can be optimized in order to practice a more effective reduction in the incidence of core hypothermia on admission at post-anesthesia care unit (PACU) [1].

The aim of this study was to estimate the incidence, predictors and outcome of core hypothermia on admission at PACU.

\section{Methods}

The institutional review board of Centro Hospitalar São João approved the study (Ethics Committee of Centro Hospitalar São João, Porto, Portugal, Chairperson Prof. Dr. Filipe N. A. S. Almeida) and informed consent was obtained preoperatively from each study patient. This prospective study was carried out in the Post-Anaesthesia Care Unit at the Hospital São João, an 1100-bed community teaching hospital in Porto, Portugal. All adult post-operative patients admitted to the PACU who underwent scheduled or emergency non-cardiac and nonintracranial surgery between 9 May 2011 and 31 May 2011 were eligible to the study.

Patients who did not provide or were incapable of providing informed consent due to intellectual or psychiatric disturbance that precluded complete cooperation, a history of alcohol or drug dependence, non-Portuguese speaking, distress or any severe pre-existing medical condition that limited objective assessment after operation or the presence of any life-threatening postoperative complication were excluded from the study.

In addition to informed consent, during the preoperative period, it was performed the MMSE [17].

The variables recorded on admission to the PACU were age, gender, body mass index (BMI), ASA physical status, preadmission comorbidities (congestive heart failure, COPD, hypertension, hyperlipidemia), and admission's temperature and pain VAS (Visual Analogic Scale). The Revised Cardiac Risk Index (RCRI) was calculated as described by Lee et al. [18] assigning one point for each of the following risk factors: high-risk surgery, ischemic heart disease, cerebrovascular disease, insulin therapy for diabetes and renal failure. Data collection included the type of anesthesia, anesthetics, intraoperative fluids, duration of anesthesia, duration of surgery, type of active warming during the surgery. Postoperatively, vital signs were recorded, as well as, length of PACU and hospital stay.

Core temperature $(\mathrm{Tc})$ was evaluated using an infrared tympanic membrane thermometer (Thermos Scan ${ }^{\circledR}$ Type 6014 Pro 3000, Welch Allyn, with accuracy of $\pm 0.03^{\circ} \mathrm{C}$ in the range of $20^{\circ} \mathrm{C}-42.2^{\circ} \mathrm{C}$-Welch Allyn Medical Products, NY, USA). This instrument was maintained and calibrated in accordance with the manufacturer's guidelines.

Patients were classified as hypothermic $\left(\mathrm{Tc}<35^{\circ} \mathrm{C}\right)$ or normothermic $\left(\mathrm{Tc} \geq 35^{\circ} \mathrm{C}\right)$.

\section{Statistical Analysis}

Descriptive analysis of variables was used to summarize data. Ordinal and continuous data found not to follow a normal distribution, based on the Kolmogorov-Smirnov test for normality of the underlying population, are presented as median and interquartile range. Normally distributed data are presented as mean and standard deviation (SD). An univariate analysis was performed to identify determinants for hypothermia using the MannWhitney $\mathrm{U}$ test to compare continuous variables and Chi-square or Fisher's exact test to compare proportions between two groups of subjects. A multiple logistic regression analysis with an odds ratio (OR) and $95 \%$ confidence interval (CI) was performed to evaluate independent predictors of hypothermia. All variables were deemed to be significant if $\mathrm{P} \leq 0.05$. Data was analyzed using SPSS for Windows version 16.0 (SPSS, Chicago, IL).

\section{Results}

A total of 357 patients, 340 were included in the study (Table 1), 17 patients were excluded: 7 patients were admitted in a surgical intensive care unit, 3 patients were incapable of providing informed consent or had a MMSE $<25,3$ patients were not submitted to surgery, 1 patient was excluded because was submitted to a neurosurgery, 1 was less than 18 years old, 1 did not speak Portuguese and 1 refused to participate.

Median $\mathrm{Tc}$ at admission was $35.3^{\circ} \mathrm{C}$ (interquartile range, $\left.34.9^{\circ} \mathrm{C}-35.7^{\circ} \mathrm{C}\right) ; 32.6^{\circ} \mathrm{C}$ was the lowest temperature registered and $37.5^{\circ} \mathrm{C}$ the highest. Incidence 
Table 1. Characteristics of patients $(n=340)$.

\begin{tabular}{|c|c|}
\hline Variable & Median and IQR or number (\%) \\
\hline Age & $57(44-6)$ \\
\hline$<65 / \geq 65$ years & $226(67) / 114(34)$ \\
\hline Male/Female & $146(43) / 194(57)$ \\
\hline Body mass index $\left(\mathrm{kg} / \mathrm{m}^{2}\right)$ & $26(23-30)$ \\
\hline \multicolumn{2}{|l|}{ ASA physical status } \\
\hline $\mathrm{I} / \mathrm{II}$ & $277(82)$ \\
\hline $\mathrm{III} / \mathrm{IV} / \mathrm{V}$ & $63(19)$ \\
\hline High risk surgery & $93(27)$ \\
\hline \multicolumn{2}{|l|}{ Heart disease } \\
\hline Ischemic & $21(6)$ \\
\hline Congestive & $20(6)$ \\
\hline Cerebrovascular disease & $9(3)$ \\
\hline Insulin therapy for diabetes & $49(14)$ \\
\hline Preoperative serum creatinine $>2 \mathrm{mg} / \mathrm{dl}$ & $17(5)$ \\
\hline \multicolumn{2}{|l|}{ RCRI } \\
\hline$\leq 2$ & $326(96)$ \\
\hline$>2$ & $15(4)$ \\
\hline COPD & $19(6)$ \\
\hline Hypertension & $141(42)$ \\
\hline Hyperlipidemia & $92(27)$ \\
\hline \multicolumn{2}{|l|}{ Magnitude of surgery } \\
\hline Minor & $35(10)$ \\
\hline Medium & $213(63)$ \\
\hline Major & $34(10)$ \\
\hline \multicolumn{2}{|l|}{ Surgical risk } \\
\hline Minor & $74(22)$ \\
\hline Medium & $232(68)$ \\
\hline Major & $34(10)$ \\
\hline \multicolumn{2}{|l|}{ Type of anesthesia } \\
\hline General & $241(71)$ \\
\hline Regional & $69(20)$ \\
\hline Combined & $30(9)$ \\
\hline Warming methods & $116(34)$ \\
\hline Fluids & $24(7)$ \\
\hline Forced-air warming technic & $111(33)$ \\
\hline Intraoperative intravenous crystalloids (L.) & $1.0(1.0-1.9)$ \\
\hline Duration of anesthesia (min) & $20(71-180)$ \\
\hline Duration of surgery (min) & $80(50-130)$ \\
\hline Core Temperature at admission & $35.3(34.9-35.7)$ \\
\hline$<35^{\circ} \mathrm{C}$ & $110(32)$ \\
\hline Length of PACU stay (hours) & $95(65-130)$ \\
\hline Length of Hospital stay (days) & $2(4-7)$ \\
\hline
\end{tabular}

IQR, Interquartil range; L., Liters; PACU, Postoperative Anesthesia Care Unit; ASA, American Society of Anesthesiologists; RCRI, Revised Cardiac Risk Index; COPD, Chronic obstructive pulmonary disease. 
of core hypothermia on the admission in PACU was $32.4 \%(95 \%$ CI, 29.8\%, 34.9). Eighty-six percent of patients had less than $36.0^{\circ} \mathrm{C}$.

Patients with hypothermia (Table 2) had more frequently age $\geq 65$ years $(42 \%$ versus $30 \%, \mathrm{P}=0.025)$, had lower BMI $(25.9 \pm 5.0$ versus $27.8 \pm 5.9, \mathrm{P}=0.001)$, were more frequently submitted to high risk surgery ( $35 \%$ versus $24 \%, \mathrm{P}=0.04$ ), had higher scores in RCRI (RCRI $>2,8 \%$ versus $3 \%, \mathrm{P}=0.019)$, had less frequently loco regional anesthesia as a unique technic (13\% versus $24 \%, \mathrm{P}=0.016)$, more frequently a combine anesthesia technic $(14 \%$ versus $7 \%, \mathrm{P}=0.011)$ and more frequently used a forced-air warming technic ( $42 \%$ versus $28 \%, \mathrm{P}=$ $0.013)$. The total amount of intravenous crystalloids administrated were higher (median of 1.7 versus $1.2 \mathrm{~L}$., $\mathrm{P}<$ 0.001 ), had a longer duration of anesthesia (median of 150 versus $102 \mathrm{~min}, \mathrm{P}<0.001$ ) and of surgery (median of 110 versus $70 \mathrm{~min}, \mathrm{P}<0.001$ ) and had higher scores for visual analogue scale of pain at PACU admission (VAS $>3$, median of 2.4 versus $1.0, \mathrm{P}=0.031$ ). Hypothermia was a risk factor for longer length postanesthesia care unit stay (65 - 125 versus $75-147$ min., $\mathrm{P}=0.009)$ and was not a predictor for longer hospital stay.

In multiple logistic regression analysis (Table 3), independent predictors of hypothermia were: age (OR 1.72, $95 \% \mathrm{CI}, 1.01-2.91, \mathrm{P}=0.045$ ), RCRI $>2$ (OR 3.18, $95 \% \mathrm{CI}, 1.05-9.64, \mathrm{P}=0.041)$, duration of anesthesia (OR 1.52, 95\% CI, $1.26-1.83, \mathrm{P}<0.001$ ) and VAS pain at PACU admission (OR 2.05, 95\% CI, $1.23-3.45, \mathrm{P}=$ $0.007)$.

Vital signs measured on admission to the PACU admission (Table 4) showed no statistically significant differences between patients with hypothermia and normothermia, except with regard to pain evaluation.

During hospital stay 2 patients died (one was hypothermic at PACU admission).

\section{Discussion}

The principal findings of this study were as follow:

Hypothermia at PACU admission was $32 \%$.

Multivariate analysis found that age, RCRI, duration of anesthesia and VAS for pain at PACU admission were independent predictors for postoperative inadvertent hypothermia.

Patients with hypothermia at PACU admission stay longer in the PACU.

The incidence of hypothermia at PACU admission was higher than in other studies that have considered the same cut-off of $35^{\circ} \mathrm{C}$ to define hypothermia $[1,12,19]$. In a previous published study performed in the same department [12], authors found a higher incidence, but the studied cohort was composed of critical care surgical patients.

The use of infrared tympanic thermometers to measure the patient's temperature has been referred as a potential limitation for temperature evaluation. Nierman et al. [20] found that comparing the "gold standard" of a thermistor in a pulmonary artery catheter to infrared tympanic, the tympanic thermometer present a bias (mean difference) of $0.1 \%-0.4 \%$ less than thermistors, situation that could overestimate hypothermia, when the measurement is made by tympanic thermometers [19]. Therefore, considering the greatest reported bias, the real incidence of hypothermia in our study could have been slightly lower. Concerning this subject, Sessler [7] stated that when infrared signals are obtained exactly from the tympanic membrane, the result is real Tc. The limitation that Sessler recognizes to this type of evaluation is that there are infrared systems that, for its size, do not allow to reach the tympanic membrane and therefore are used only in aural canal performing an insufficiently accurate measure for clinical use.

The independent predictors of hypothermia found in this study were age, the RCRI, the duration of anesthesia and pain at PACU admission.

Previous studies have shown that older patients [21-23] had an increased risk for hypothermia and although the British National Institute for Health and Clinical Excellence (NICE) [2] assume that age is not an important risk factor for the incidence of hypothermia, in this study, age appears as an independent risk factor for postoperative inadvertent hypothermia. This is an important finding since data suggests that older people (over 60 years) have lower temperatures after 3 hours of surgery and at PACU arrival and that some consequences of hypothermia are more severe for older people, especially morbid cardiac events.

Hypothermia may have an effect on the pain experienced by patients who undergo surgery and the link between hypothermia and pain is not well understood. Studies in animals and humans offer conflicting evidence as to how hypothermia affects pain and opioid disposition. In the studies of Kurz [24] and Wongprasartsuk [25] pain scores were identical in two subgroups of patients concerning different strategies to prevent postoperative hypothermia. In another study with the objective of clarifying whether perioperative developed hypothermia affects postoperative opioid requirements, Persson et al. [26] found that there were no postoperative differences in analgesic requirements or pain intensity between normothermic and hypothermic patients. According to our results we can speculate whether it is pain that increases the incidence of hypothermia or if hypothermia leads to more pain postoperatively, but for our knowledge this is 
Table 2. Analysis of categorical and continuous variables of core hypothermia.

\begin{tabular}{|c|c|c|c|}
\hline Variable & $>35^{\circ} \mathrm{C}$ & $\leq 35^{\circ} \mathrm{C}$ & P-value \\
\hline Age & $54.6 \pm 15.6 / 56(44-66)$ & $57.7 \pm 16.7 / 60(47-71)$ & 0.053 \\
\hline$<65 / \geq 65$ & $162(70) / 68(30)$ & $64(58) / 46(42)$ & 0.025 \\
\hline Male/Female & $91(40) / 139(60)$ & $55(50) / 55(50)$ & 0.069 \\
\hline Body Mass Index $\left(\mathrm{Kg} / \mathrm{m}^{2}\right)$ & $27.8 \pm 5.9 / 27(24-31)$ & $25.9 \pm 5.0 / 27(22-28)$ & 0.001 \\
\hline ASA physical status & & & 0.854 \\
\hline $\mathrm{I} / \mathrm{II}$ & $188(82)$ & $89(81)$ & \\
\hline III/IV/V & $42(18)$ & $21(19)$ & \\
\hline High risk surgery & $55(24)$ & $38(35)$ & 0.040 \\
\hline Ischemic heart disease & $11(5)$ & $10(9)$ & 0.123 \\
\hline Congestive heart disease & $11(5)$ & $9(8)$ & 0.213 \\
\hline Cerebrovascular disease & $7(3)$ & $2(2)$ & 0.510 \\
\hline Insulin therapy for diabetes & $36(16)$ & $13(12)$ & 0.346 \\
\hline Serum creatinine $>2 \mathrm{mg} / \mathrm{dl}$ & $9(4)$ & $8(7)$ & 0.184 \\
\hline RCRI & & & 0.019 \\
\hline$\leq 2$ & $224(97)$ & $102(93)$ & \\
\hline$>2$ & $6(3)$ & $9(8)$ & \\
\hline Hypertension & $96(42)$ & $45(41)$ & 0.884 \\
\hline Hyperlipidemia & $64(28)$ & $28(26)$ & 0.646 \\
\hline COPD & $12(5)$ & $7(6)$ & 0.667 \\
\hline Magnitude of surgery & & & 0.162 \\
\hline Minor & $24(10)$ & $11(10)$ & \\
\hline Medium & $151(66)$ & $62(56)$ & \\
\hline Major & $55(24)$ & $37(34)$ & \\
\hline Surgical risk & & & 0.106 \\
\hline Minor & $57(25)$ & $17(16)$ & \\
\hline Medium & $153(66)$ & $79(72)$ & \\
\hline Major & $20(9)$ & $14(13)$ & \\
\hline Type of anesthesia & & & 0.011 \\
\hline General & $160(70)$ & $81(74)$ & \\
\hline Locoregional & $55(24)$ & $14(13)$ & \\
\hline Combined & $17(7)$ & $15(14)$ & \\
\hline Warming methods & $62(28)$ & $48(41)$ & 0.010 \\
\hline Fluids & $17(7)$ & $7(6)$ & 0.729 \\
\hline Forced-air warming technic & $65(28)$ & $46(42)$ & 0.013 \\
\hline Intraoperative intravenous crystalloids (L.) & $1.236 \pm 0.910(1.000-1.125)$ & $1.724 \pm 1.246(1.000-2.380)$ & $<0.001$ \\
\hline Duration of anesthesia (min.) & $120 \pm 75 / 102(60-150)$ & $163 \pm 81 / 150(99-210)$ & $<0.001$ \\
\hline Duration of surgery (min.) & $88 \pm 63 / 70(40-120)$ & $120 \pm 69 / 110(60-162)$ & $<0.001$ \\
\hline Core Temperature at admission $<35^{\circ} \mathrm{C}$ & $35.6 \pm 0.4 / 35.6(35.3-35.9)$ & $34.6 \pm 0.5 / 34.6(34.4-34.9)$ & $<0.001$ \\
\hline Length of PACU stay (min.) & $106 \pm 92 / 90(65-125)$ & $137 \pm 121 / 106(75-147)$ & 0.009 \\
\hline Length of Hospital stay (days) & $7.2 \pm 12.0 / 4(2-7)$ & $8.3 \pm 14.1 / 5(2-8)$ & 0.167 \\
\hline VAS $>3$ & $2.22 \pm 2.74 / 1(0.0-4.0)$ & $2.84 \pm 2.90 / 2.4(0.0-5.0)$ & 0.031 \\
\hline
\end{tabular}

IQR, Interquartil range; L., Liters; RCRI, Revised Cardiac Risk Index; PACU, Postoperative Anesthesia Care Unit; VAS, Visual Analogue Scale (for pain). 
Table 3. Predictors of core hypothermia by multiple logistic regression.

\begin{tabular}{|c|c|c|c|c|}
\hline Variable & Odds ratio $(95 \% \mathrm{CI})$ & P-value & Odds ratio $(95 \% \mathrm{CI})$ & P-value \\
\hline Age $\geq 65$ & $1.71(1.07-2.75)$ & 0.026 & $1.72(1.01-2.91)$ & 0.045 \\
\hline Body Mass Index $\left(\mathrm{Kg} / \mathrm{m}^{2}\right)$ & $0.94(0.89-0.98)$ & 0.005 & & \\
\hline High risk surgery & $1.68(1.02-2.76)$ & 0.041 & & \\
\hline \multicolumn{5}{|l|}{ RCRI } \\
\hline $\mathrm{RCRI} \leq 2$ & 1 & & 1 & \\
\hline RCRI $>2$ & $3.33(1.15-9.60)$ & 0.026 & $3.18(1.05-9.64)$ & 0.041 \\
\hline General anesthesia only & $2.16(1.14-4.08)$ & 0.018 & & \\
\hline Combined anesthesia & $2.26(1.06-4.82)$ & 0.034 & & \\
\hline Warming methods & $1.84(1.15-2.96)$ & 0.011 & & \\
\hline Intravenous crystalloids (liters) & $1.58(1.24-2.02)$ & $<0.001$ & & \\
\hline Duration of anesthesia (hours) & $1.50(1.25-1.79)$ & $<0.001$ & $1.52(1.26-1.83)$ & $<0.001$ \\
\hline Duration of surgery (hours) & $1.52(1.24-1.87)$ & $<0.001$ & & \\
\hline VAS $>3$ & $1.86(1.16-3.00)$ & 0.010 & $2.05(1.23-3.45)$ & 0.007 \\
\hline
\end{tabular}

RCRI, Revised Cardiac Risk Index; VAS, Visual Analogue Scale (for pain).

Table 4. Vital signs.

\begin{tabular}{|c|c|c|c|c|}
\hline Variable & Total & Normothermic & Hypothermic & P-value \\
\hline $\mathrm{SBP}(\mathrm{mmHg})$ & $129 \pm 25$ & $131 \pm 24$ & $127 \pm 27$ & 0.161 \\
\hline DBP (mmHg) & $69 \pm 14$ & $70 \pm 13$ & $69 \pm 15$ & 0.512 \\
\hline HR (bpm) & $74 \pm 16$ & $75 \pm 16$ & $74 \pm 16$ & 0.630 \\
\hline $\mathrm{O}_{2}$ Sat $(\%)$ & $96 \pm 3.4$ & $96 \pm 3$ & $97 \pm 4$ & 0.162 \\
\hline $\mathrm{VAS}>3$ & 110 & $64(28)$ & $46(42)$ & 0.010 \\
\hline
\end{tabular}

SBP, systolic blood pressure; DBP, diastolic blood pressure; HR, heart rate; $\mathrm{O}_{2}$ Sat, oxygen saturation; VAS, Visual Analogue Scale (for pain).

the first study in which pain appears as an independent risk factor for inadvertent hypothermia after surgery.

Patients at high risk of perioperative hypothermia are generally those who are at increased risk of a morbid cardiac event [2] it is why it is not unexpected that in our study patients with a higher RCRI had increased risk of inadvertent hypothermia.

What we may conclude is that patients that are at higher risk for hypothermia may also be at higher risk of developing cardiac events what is agreeing with Frank et al. [27] that concluded in their study that in patients with cardiac risk factors the perioperative maintenance of normothermia is associated with a reduced incidence of morbid cardiac events.

According to Putzu et al. [8], independently of anesthesia technic surgical patients always develop perioperative hypothermia when the surgical procedure lasts more than 30 minutes. In the present study patients with inadvertent hypothermia at PACU admission had longer anesthesia duration but differing with the study of Kongsayreepong et al. [1] duration of anesthesia was not considered an independent predictor for hypothermia.

In our study patients with hypothermia at PACU admission stayed longer in the PACU what is in 
agreement with the British National Institute for Health and Clinical Excellence [2] in which are reported that although four of the six studies showed that hypothermic patients did not spend a significantly longer time in PACU, in the meta-analysis of the study results a weighted mean difference of 3.24 (95\% CI, 0.01, 6.48) was found.

Also, there was found in this study a positive risk relation between inadvertent hypothermia and the utilization of general anesthesia technic performed as a single technic or combined technic when compared to locorregional anesthesia only, this relation was not considered sufficient to be considered an independent risk factor for hypothermia as indicated by others [12].

Forced air warmers are one of the most effective means of warming a patient [28], and there are significant benefits associated with forced-air warming [29] and extra warming with forced air (compared to routine thermal care) may be effective in reducing the incidence of surgical wound infections [24] and postoperative cardiac complications [27]. In our study, the use of warming methods was not an independent protective factor as previously reported $[1,30,31]$. Indeed the use of warming methods was even a risk factor and it should be noted that the Odds Ratio of 1.84 in the univariate analysis (for the use of warming methods) could be explained by the fact that these warming methods were used more frequently in patients with an increased risk for inadvertent hypothermia.

We concluded that the amount of intravenous intraoperative crystalloids was a risk factor for developing hypothermia on arrival at PACU; this was already identified in previous studies $[21,22]$. In fact the infusion of crystalloid solutions at room temperature may significantly contribute to intraoperative hypothermia because warming fluids to core temperature requires body heat. Several studies had demonstrated that infusion of warmed fluids helps in the prevention of hypothermia and reduce the incidence of postoperative shivering $[32,33]$.

In our study, we did not found a relationship between ASA physical status and core hypothermia. Although, others have claimed that ASA grade greater than ASA I is a risk factor for perioperative hypothermia and that the risk increases with ASA grade [2].

Of the variables considered in this study, the BMI was a protective variable for core hypothermia, similarly to what has already been indicated by other studies[1]. In the National Institute for Health and Clinical Excellence Guideline [2] it is admitted that increased body weight may have a small protective effect on the incidence of perioperative hypothermia in PACU, but the evidence for body weight and body fat in thermic regulation is inconsistent.

As limitations of our study we may indicate the lack of information about operating room temperature, the absence of data about how often had been used warmed intraoperative fluids and what methods were used to warm them and temperature of patients before surgery. These variables may have had important implications in the observed results.

Despite these limitations, the results showed that postoperative inadvertent hypothermia is still frequent. Also, BMI may be protective, high-risk surgery, the technique of combined epidural and general anesthesia, using warming methods and the amount of intravenous crystalloids tended to be risk factors. Age, RCRI, duration of anesthesia and VAS for pain were significant risk factors for inadvertent hypothermia at PACU admission.

Although the adverse effects of hypothermia in surgical patients are well known, incidence remains higher. The knowledge of predictors of perioperative hypothermia may be critical to its prevention and to optimize clinical care provided.

\section{Conclusions}

Hypothermia at PACU admission was $32 \%$.

IPH was associated with a longer stay in the PACU. Age, comorbidities duration of anesthesia and pain at PACU admission were considered independent predictors for IPH.

\section{REFERENCES}

[1] S. Kongsayreepong, C. Chaibundit, J. Chadpaibool, C. Komoltri, S. Suraseranivongse, P. Suwannanonda, E. O. Raksamanee, P. Noocharoen, A. Silapadech, S. Parakkamodom, et al., "Predictor of Core Hypothermia and the Surgical Intensive Care Unit," Anesthesia \& Analgesia, Vol. 96, No. 3, 2003, pp. 826-833. doi:10.1213/01.ANE.0000048822.27698.28

[2] National Institute for Health and Clinical Excellence Guideline, "Inadvertent Perioperative Hypothermia," 2008 http://www.nice.org.uk/nicemedia/pdf/CG65Guidance.pd f

[3] L. Reynolds, J. Beckmann and A. Kurz, "Perioperative Complications of Hypothermia," Best Practice \& Research Clinical Anaesthesiology, Vol. 22, No. 4, 2008, pp. 645-657. doi:10.1016/j.bpa.2008.07.005

[4] DI Sessler, "Perioperative Heat Balance," Anesthesiology, Vol. 92, No. 2, 2000, pp. 578-596. doi:10.1097/00000542-200002000-00042

[5] D. I. Sessler, "New Surgical Thermal Management Guidelines," Lancet, Vol. 374, No. 9695, 2009, pp. 10491050. doi:10.1016/S0140-6736(09)61686-X

[6] A. Kurz, "Physiology of Thermoregulation," Best Practice \& Research Clinical Anaesthesiology, Vol. 22, No. 4, 2008, pp. 627-644. doi:10.1016/j.bpa.2008.06.004

[7] D. I. Sessler, "Temperature Monitoring and Perioperative 
Thermoregulation," Anesthesiology, Vol. 109, No. 2, 2008, pp. 318-338. doi:10.1097/ALN.0b013e31817f6d76

[8] M. Putzu, A. Casati, M. Berti, G. Pagliarini and G. Fanelli, "Clinical Complications, Monitoring and Management of Perioperative Mild Hypothermia: Anesthesiological Features," Acta BioMedica, Vol. 78, No. 3, 2007, pp. 163-169.

[9] A. Torossian, "Survey on Intraoperative Temperature Management in Europe," European Journal of Anaesthesiology, Vol. 24, No. 8, 2007, pp. 668-675. doi:10.1017/S0265021507000191

[10] S. R. Insler and D. I. Sessler, "Perioperative Thermoregulation and Temperature Monitoring," Anesthesiology Clinics, Vol. 24, No. 4, 2006, pp. 823-837. doi:10.1016/i.atc.2006.09.001

[11] C. B. Biazzotto, M. Brudniewski, A. P. Schmidt and J. O. Auler Junior, "Perioperative Hypothermia," Revista Brasileira de Anestesiologia, Vol. 56, No. 1, 2006, pp. 89106. doi:10.1590/S0034-70942006000100012

[12] F. J. Abelha, M. A. Castro, A. M. Neves, N. M. Landeiro and C. C. Santos, "Hypothermia in a Surgical Intensive Care Unit," BMC Anesthesiology, Vol. 5, 2005, p. 7. doi:10.1186/1471-2253-5-7

[13] S. Rajagopalan, E. Mascha, J. Na and D. I. Sessler, "The Effects of Mild Perioperative Hypothermia on Blood Loss and Transfusion Requirement," Anesthesiology, Vol. 108, No. 1, 2008, pp. 71-77. doi:10.1097/01.anes.0000296719.73450.52

[14] R. Tiruvoipati, K. Ong, H. Gangopadhyay, S. Arora, I. Carney and J. Botha, "Hypothermia Predicts Mortality in Critically Ill Elderly Patients with Sepsis," BMC Geriatrics, Vol. 10, 2010, p. 70. doi:10.1186/1471-2318-10-70

[15] J. Andrzejowski, J. Hoyle, G. Eapen and D. Turnbull, "Effect of Prewarming on Post-Induction Core Temperature and the Incidence of Inadvertent Perioperative Hypothermia in Patients Undergoing General Anaesthesia," British Journal of Anaesthesia, Vol. 101, No. 5, 2008, pp. 627-631. doi:10.1093/bja/aen272

[16] S. M. Frank, H. K. El-Rahmany, C. G. Cattaneo and R. A. Barnes, "Predictors of Hypothermia during Spinal Anesthesia," Anesthesiology, Vol. 92, No. 5, 2000, pp. 13301334. doi:10.1097/00000542-200005000-00022

[17] M. F. Folstein, S. E. Folstein and P. R. McHugh, "MiniMental State: A Practical Method for Grading the Cognitive State of Patients for the Clinician," Journal of Psychiatric Research, Vol. 12, No. 3, 1975, pp. 189-198. doi:10.1016/0022-3956(75)90026-6

[18] T. H. Lee, E. R. Marcantonio, C. M. Mangione, E. J. Thomas, C. A. Polanczyk, E. F. Cook, D. J. Sugarbaker, M. C. Donaldson, R. Poss, K. K. Ho, et al, "Derivation and Prospective Validation of a Simple Index for Prediction of Cardiac Risk of Major Noncardiac Surgery," Circulation, Vol. 100, No. 10, 1999, pp. 1043-1049. doi:10.1161/01.CIR.100.10.1043

[19] D. Karalapillai, D. A. Story, P. Calzavacca, E. Licari, Y. L. Liu and G. K. Hart, "Inadvertent Hypothermia and Mortality in Postoperative Intensive Care Patients: Ret- rospective Audit of 5050 Patients," Anaesthesia, Vol. 64, No. 9, 2009, pp. 968-972.

doi:10.1111/j.1365-2044.2009.05989.x

[20] D. M. Nierman, “Tools that we use, "If You Can't Measure It, You Can't Manage It," Critical Care Medicine, Vol. 35, No. 1, 2007, pp. 312-313. doi:10.1097/01.CCM.0000251297.60407.DB

[21] H. L. Bush Jr., L. J. Hydo, E. Fischer, G. A. Fantini, M. F. Silane and P. S. Barie, "Hypothermia during Elective Abdominal Aortic Aneurysm Repair: The High Price of Avoidable Morbidity," Journal of Vascular Surgery, Vol. 21, No. 3, 1995, pp. 392-400; Discussion pp. 400-392.

[22] G. J. Slotman, E. H. Jed and K W. Burchard, "Adverse Effects of Hypothermia in Postoperative Patients," The American Journal of Surgery, Vol. 149, No. 4, 1985, pp. 495-501. doi:10.1016/S0002-9610(85)80046-5

[23] S. Roberts, D. M. Bolton and M. L. Stoller, "Hypothermia Associated with Percutaneous Nephrolithotomy," Urology, Vol. 44, No. 6, 1994, pp. 832-835. doi:10.1016/S0090-4295(94)80166-5

[24] A. Kurz, D. I. Sessler and R. Lenhardt, Study of Wound Infection and Temperature Group, "Perioperative Normothermia to Reduce the Incidence of Surgical-Wound Infection and Shorten Hospitalization," The New England Journal of Medicine, Vol. 334, No. 19, 1996, pp. 1209. 1215. doi:10.1056/NEJM199605093341901

[25] P. Wongprasartsuk, A. Konstantatos and R. McRae, "The Effect of Forced Air Warming on Postoperative Oxygen Consumption and Temperature in Elective Orthopaedic Surgery," Anaesthesia and Intensive Care Journal, Vol. 26, No. 3, 1998, pp. 267-271.

[26] K. Persson and J. Lundberg, "Perioperative Hypothermia and Postoperative Opioid Requirements," European Journal of Anaesthesiology, Vol. 18, No. 10, 2001, pp. 679686.

[27] S. M. Frank, L. A. Fleisher, M. J. Breslow, M. S. Higgins, K. F. Olson, S. Kelly and C. Beattie, "Perioperative Maintenance of Normothermia Reduces the Incidence of Morbid Cardiac Events. A Randomized Clinical Trial," Japan Automobile Manufacturers Association, Vol. 277, No. 14, 1997, pp. 1127-1134.

[28] S. R. Hart, B. Bordes, J. Hart, D. Corsino and D. Harmon, "Unintended Perioperative Hypothermia," The Ochsner Journal, Vol. 11, No. 3, 2011, pp. 259-270.

[29] S. Moola and C. Lockwood, "Effectiveness of Strategies for the Management and/or Prevention of Hypothermia within the Adult Perioperative Environment," International Journal of Evidence-Based Healthcare, Vol. 9, No. 4, 2011, pp. 337-345. doi:10.1111/j.1744-1609.2011.00227.x

[30] N. El-Gamal, N. El-Kassabany, S. M. Frank, R. Amar, H. A. Khabar, H. K. El-Rahmany and A. S. Okasha, "AgeRelated Thermoregulatory Differences in a Warm Operating Room Environment (Approximately 26 Degrees C)," Anesthesia \& Analgesia, Vol. 90, No. 3, 2000, pp. 694698. doi:10.1097/00000539-200003000-00034

[31] S. M. Vanni, J. R. Braz, N. S. Modolo, R. B. Amorim and G. R. Rodrigues Jr., "Preoperative Combined with Intra- 
operative Skin-Surface Warming Avoids Hypothermia Caused by General Anesthesia and Surgery," Journal of Clinical Anesthesia, Vol. 15, No. 2, 2003, pp. 119-125. doi:10.1016/S0952-8180(02)00512-3

[32] Y. Camus, E. Delva, S. Cohen and A. Lienhart, "The Effects of Warming Intravenous Fluids on Intraoperative Hypothermia and Postoperative Shivering during Prolonged Abdominal Surgery," Acta Anaesthesiologica Scan- dinavica, Vol. 40, No. 7, 1996, pp. 779-782. doi:10.1111/j.1399-6576.1996.tb04532.x

[33] C. E. Smith, R. Desai, V. Glorioso, A. Cooper, A. C. Pinchak and K. F. Hagen, "Preventing Hypothermia: Convective and Intravenous Fluid Warming versus Convective Warming Alone," Journal of Clinical Anesthesia, Vol. 10, No. 5, 1998, pp. 380-385. doi:10.1016/S0952-8180(98)00049-X 